\title{
Editorial Novel Stimulation Approaches with ECT: Why Everything Old May be New Again
}

\author{
Zafiris J Daskalakis*, and Yinming Sun' \\ 'Department of Psychiatry, Temerty Centre for Therapeutic Brain Intervention, Campbell Family Research Institute, Centre for Addiction and \\ Mental Health, University of Toronto, Toronto, ON, Canada
}

Neuropsychopharmacology (20 I7) 42, I56 I-I562; doi:| 0.1038/npp.2017.42

Major Depressive Disorder (MDD) results in significant morbidity, functional impairment, and mortality. By the year 2020 , depression is estimated to be second only to heart disease in magnitude of disease burden. Approximately $30-40 \%$ of patients with MDD have treatment resistant depression (TRD), which is conventionally defined as a failure to respond to two adequate medication trials or as a relapse during treatment.

Electroconvulsive therapy (ECT) is currently the most effective intervention for TRD. Bitemporal and bifrontal ECT involve bilateral stimulation of the temporal and frontal regions, respectively. Right unilateral (RUL) ECT involves stimulation delivered to the right temporal area and vertex. In a recent and large, multicentre, double-blind randomized clinical trial involving 230 patients with depression, Kellner et al, (2010) reported that all three electrode placements resulted in high rates of remission with ECT that did not differ between the treatment groups (55\% for RUL, $61 \%$ for bifrontal, and $64 \%$ with bitemporal). In addition, it was reported that bitemporal ECT resulted in the most rapid clinical response.

ECT is also an effective treatment for suicidality. Suicidality is a significant public health problem that continues to grow despite tremendous prevention and treatment efforts. In 2012, over 804000 people worldwide committed suicide. TRD is often accompanied by suicidal ideation and $15-20 \%$ of patients with MDD/TRD end their lives by suicide. The World Health Organization recently proposed that new research into suicide prevention treatments be advanced. In this regard, there are few treatment options available for suicidality in depressed patients. Research has demonstrated the effective use of ECT for patients with TRD and suicidal ideation. For example, Kellner et al, (2005) evaluated ECT in 131 patients who reported suicidal ideation. ECT produced a remission or near

\footnotetext{
*Correspondence: Dr ZJ Daskalakis, Department of Psychiatry, Temerty Chair in Therapeutic Brain Intervention, Mood and Anxiety Division, Campbell Family Research Institute, Centre for Addiction and Mental Health, 1001 Queen Street West, Toronto, ON M6) IH4, Canada, Tel: +4165358501 ext. 34319, Fax: +416583 I358,

E-mail: Jeff.Daskalakis@camh.ca
}

remission of suicidal ideation in $61.1 \%$ of patients after only 2 weeks of treatment and in $80.9 \%$ of patients at the completion of treatment.

However, ECT is complicated by its negative stigma and adverse cognitive effects. ECT is so negatively stigmatized that only $1 \%$ of patients with TRD are willing to pursue ECT. Also, regardless of treatment delivery, ECT results in significant post-ictal disorientation, and anterograde and retrograde amnesia. Disorientation immediately after ECT can last up to $40 \mathrm{~min}$, regardless of electrode placement, which lengthens the procedure and causes patients distress. Anterograde amnesia, the inability to learn and retain new information, can appear after the first ECT treatment and persist for up to several months. Retrograde amnesia, the inability to recall past personal and impersonal memories, remains the most serious ECT cognitive adverse effect. Retrograde amnesia is more severe with bilateral ECT (ie, bifrontal or bitemporal) than RUL ECT, with high compared to low dosages, and with brief pulse width relative to ultrabrief pulse width (Sackeim et al, 2008). As recently highlighted by the US FDA, the extent of anterograde and retrograde amnesia, and the degree to which memory remains impaired post-ECT is a significant problem for patients and their families. Numerous patients refuse ECT because of these adverse cognitive effects.

MST is an alternative form of convulsive therapy that also involves the induction of a seizure to achieve therapeutic response. However, the induction of a seizure occurs through the use of high-frequency transcranial magnetic stimulation rather than high-frequency transcranial electrical stimulation. Rapid, high intensity, time varying magnetic field is able to pass into the brain unimpeded and without being shunted by non-conducting material (ie, skull). This allows MST to control the seizure spread by stimulating the brain with a focal electric field based on the geometry of the stimulating coil. Specifically, compared to RUL ultrabrief pulse ECT (RUL-UB ECT), the electric field induced by MST can be 5-10 times more focal. MST has been shown to produce significant therapeutic effects on both depressive symptoms (Kayser et al, 2015) and suicidality (Sun et al, 2016). 
In this issue of Neuropsychopharmacology, Lee and colleagues have provided compelling evidence for the potential of using electric field simulations as a way to personalize convulsive therapy and reduce its side effects (Lee et al, 2017). In this study, they simulated the electric field maps produced by four different ECT electrode configurations (bitemporal, bifrontal, RUL, and frontomedial) and one form of MST (cap coil on vertex) in realistic head models of non-human primates based on individual MRI images. On the basis of the simulation, RUL ECT can produce a seizure by exposing the brain to similar levels of electric field as MST when the current amplitude is titrated for the individual. Titrating the current amplitude needed to reach a seizure for individuals rather than using a fixed value reduces excessive stimulation of the brain (Peterchev et al, 2015). The current study builds upon that knowledge by showing that lower seizure threshold leads to smaller and more focally stimulated brain volume. Furthermore, titrating the stimulation amplitude for both ECT and MST reduced the variability associated with the stimulated brain volume across individuals. On the basis of such findings, it is conceivable that seizure thresholds may be predicted for individuals in a clinical setting based on the electric field generated in their head model for a given stimulation setup.

While the findings of Lee and colleagues are very encouraging, several additional studies are needed before such an approach for convulsive therapy can be adopted into routine clinical practise. First, the accuracy and reliability of using a simulation model for determining seizure threshold should be quantified. Second, the efficacy and cognitive adverse effects of using amplitude titrated ECT should be examined in subjects with TRD. Finally, since RUL-UB ECT is currently the gold standard in terms of efficacy and cognitive side effects, a direct comparison of the amplitudetitrated and standard versions of RUL-UB ECT is required to establish non-inferiority of the approach.

A long-standing argument in the field is why modify ECT when it works exceptionally well for TRD. The answer to this is simple. While very effective, ECT is underutilized as patients and clinicians often consider it a treatment of last resort-largely due to the cognitive side effects that are substantial with bilateral ECT. Alternative treatment approaches that involve more focal and personalized ECT or MST may result in some compromised efficacy, but with fewer cognitive side effects, these new approaches are anticipated to be far more acceptable. Thus, the innovative study by Lee and colleagues should provide investigators the impetus to further develop new forms of seizure treatment, one of the most effective approaches for the most severe TRD patients.

\section{FUNDING AND DISCLOSURE}

In the last 3 years, ZJD has received research and equipment in-kind support for an investigator-initiated study through Brainsway Inc and Magventure Inc. ZJD has also received monies for participation on an advisory board from Sunovion Inc. Finally, ZJD owns $>\$ 10000$ (CAD) in stock of Biogen Inc. The remaining author declares no conflict of interest.

\section{REFERENCES}

Kayser S, Bewernick BH, Matusch A, Hurlemann R, Soehle M, Schlaepfer TE (2015). Magnetic seizure therapy in treatmentresistant depression: clinical, neuropsychological and metabolic effects. Psychol Med 45: 1073-1092.

Kellner CH, Fink M, Knapp R, Petrides G, Husain M, Rummans T et al (2005). Relief of expressed suicidal intent by ECT: a consortium for research in ECT study. Am J Psychiatry 162: 977-982.

Kellner CH, Knapp R, Husain MM, Rasmussen K, Sampson S, Cullum $M$ et al (2010). Bifrontal, bitemporal and right unilateral electrode placement in ECT: randomised trial. Br J Psychiatry 196: 226-234.

Lee WH, Lisanby SH, Laine AF, Peterchev AV (2017). Minimum electric field exposure for seizure induction with electroconvulsive therapy and magnetic seizure therapy. Neuropsychopharmacology (this issue).

Peterchev AV, Krystal AD, Rosa MA, Lisanby SH (2015). Individualized Low-Amplitude Seizure Therapy: Minimizing Current for Electroconvulsive Therapy and Magnetic Seizure Therapy. Neuropsychopharmacology 40: 2076-2084.

Sackeim HA, Prudic J, Nobler MS, Fitzsimons L, Lisanby SH, Payne $\mathrm{N}$ et al (2008). Effects of pulse width and electrode placement on the efficacy and cognitive effects of electroconvulsive therapy. Brain Stimul 1: 71-83.

Sun Y, Farzan F, Mulsant BH, Rajji TK, Fitzgerald PB, Barr MS et al (2016). Indicators for Remission of Suicidal Ideation Following Magnetic Seizure Therapy in Patients With Treatment-Resistant Depression. JAMA Psychiatry 73: 337-345. 